\title{
ADSORCIÓN FÍSICA SOBRE SÓLIDOS: ASPECTOS TERMODINÁMICOS
}

\author{
Juan Miguel Arias, Elihu Paternina y Daniel Barragán* \\ Departamento de Química, Facultad de Ciencias, Universidad Nacional de Colombia, Carrera 30 No 45-03, Bogotá, Colombia
}

Recebido em 4/6/08; aceito em 7/11/08; publicado na web em 28/4/09

\begin{abstract}
PHYSICAL ADSORPTION ON SOLIDS: THERMODYNAMIC ASPECTS. A thermodynamic formalism based on the Gibbs Dividing Surface (GDS) for the description of a solid-fluid interface is presented, so that the adsorption layer is understand as a phase and the adsorption process as the transference of components between a 3-dimensional phase and a 2-dimensional one. Using a state equation derived from the Henry's Law, we shall show how the Langmuir isotherm is deduced from de Gibbs isotherm. The GDS is useful also for understanding the release of heat by a system as the adsorption occurs.
\end{abstract}

Keywords: physical adsorption; Gibbs isotherm; isosteric enthalpy.

\section{INTRODUCCIÓN}

El formalismo termodinámico clásico desarrollado para la descripción de los fenómenos de adsorción se basa en el concepto de equilibrio y en el modelo de la superficie divisora de Gibbs. Si bien el modelo de Gibbs tiene ventajas importantes, particularmente un formalismo simple que considera la capa adsorbida como una fase, también exhibe algunas particularidades, como: la utilización de cantidades de exceso relativas cuyo significado puede ser difícil de comprender, esencialmente cuando se intentan relacionar estas magnitudes con las cantidades adsorbidas en la interfase y la ubicación del plano de Gibbs, con volumen cero, sobre la superficie del sólido.

\section{GENERALIDADES}

La adsorción es el enriquecimiento (adsorción positiva o simplemente adsorción) o agotamiento (adsorción negativa) de uno o más componentes en una interfase o capa interfacial. En este proceso la especie que se va a adsorber es el adsortivo, que en el estado adsorbido recibe el nombre de adsorbato. Cuando una de las fases involucradas en la adsorción es sólida, a esta se le llama adsorbente. ${ }^{1,2}$

La adsorción es física cuando la naturaleza de las interacciones moleculares entre el adsorbente y el adsorbato es de corto alcance, por esto a procesos que promuevan el enriquecimiento de un componente en una interfase debido a fuerzas de largo alcance, como son las fuerzas gravitacionales (fenómenos de sedimentación), no se les considera adsorción. ${ }^{3}$

Una de las mayores dificultades cuando se plantean modelos para describir cuantitativamente los fenómenos de adsorción, es el desconocimiento de cómo varían las concentraciones de las especies entre la superficie del sólido y el seno de la fase fluida. G. W. Gibbs en 1877 introdujo el concepto de exceso de superficie haciendo referencia a la cantidad de cada componente en la superficie ó simplemente la cantidad adsorbida. ${ }^{2}$ Para formalizar esta cantidad Gibbs planteó estudiar el proceso de adsorción en un sistema de referencia dividido en dos zonas por una superficie imaginaria, la superficie divisoria de Gibbs o GDS, de volumen cero, la cual es paralela a la superficie del adsorbente. El sistema de referencia ocupa el mismo volumen que el sistema real y las concentraciones de cada uno de los componentes en solución son constantes en función de la distancia desde el seno de

*e-mail: dabarraganra@bt.unal.edu.co la fase fluida hasta la GDS. ${ }^{1,45}$ En la Figura 1a se ilustra el perfil de concentración de una mezcla binaria en un sistema con capa interfacial de volumen diferente de cero, y en la Figura $1 \mathrm{~b}$ el correspondiente modelo de referencia de Gibbs con GDS.

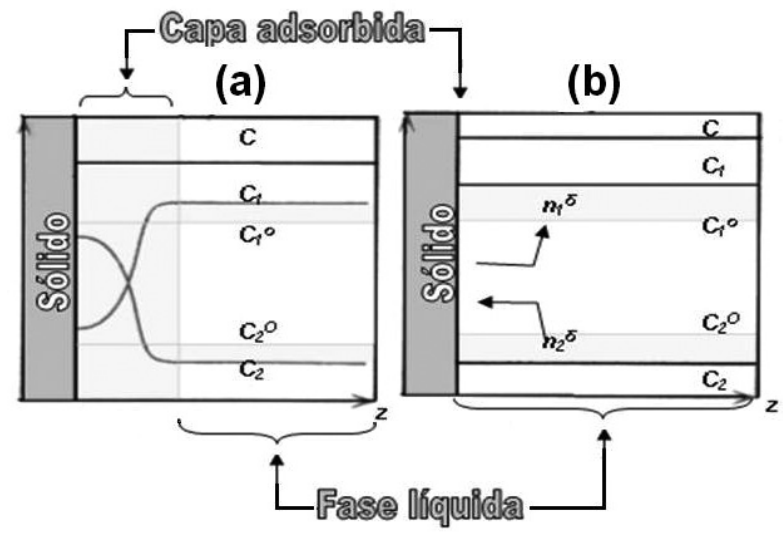

Figura 1. Perfiles de concentración en un sistema sólido/líquido (adsorción competitiva entre mezclas binarias o solución). (a) Adsorción en la capa interfacial de dos componentes con volúmenes moleculares y áreas de sección transversal similares. (b) Modelo de referencia de Gibbs donde la GDS coincide con la superficie de adsorción real del sólido. C es la concentración total de la fase fluida, $C_{1}{ }_{1}^{0} y C_{2}{ }^{0}$ son las concentraciones iniciales de cada componente, $C_{1}$ y $C_{2}$ son las concentraciones de equilibrio. Las zonas sombreadas corresponden a los cambios en la concentración del sistema. En este modelo, la cantidad de exceso de superficie $\left(n^{\sigma}\right)$ representa la cantidad adsorbida del componente en estudio en el sistema de referencia

Para el sistema de referencia Gibbs definió los potenciales termodinámicos de superficie, $\boldsymbol{P}^{\sigma}$, como aquellos correspondientes a la fase superficial GDS, de la siguiente manera

$\boldsymbol{P}^{\sigma}=\boldsymbol{P}-\boldsymbol{P}^{f}-\boldsymbol{P}^{s}$

donde $\boldsymbol{P}$ es para el sistema total de adsorción, $\boldsymbol{P}^{s}$ para el adsorbente y $\boldsymbol{P}^{f}$ para la fase fluida.

Antes de continuar con el desarrollo del formalismo termodinámico de Gibbs para los fenómenos de adsorción, es necesario definir las siguientes cantidades: 
Concentración de exceso superficial, $\Gamma$, definida como la cantidad de exceso de superficie por unidad de área,

$\Gamma=n^{\sigma} / A$

donde $A$ es para el área superficial y $\mathrm{n}^{\sigma}$ es la cantidad de componente adsorbida en la GDS.

Área específica de superficie, $a_{s p}$, definida como el área superficial, $A$, por unidad de masa del adsorbente, $m^{s}$,

$a_{s p}=A / m^{s}$

Cantidad de exceso de superficie específica, $\mathrm{a}_{\mathrm{sp}}^{\sigma}$, la cual expresa la cantidad de exceso de cada componente en la superficie por unidad de masa de adsorbente

$a_{s p}^{\sigma}=n^{\sigma} / m^{s}$

En el equilibrio la cantidad de exceso de superficie específica es función de la presión, $p$, o concentración de equilibrio, $C$, de cada componente en la fase fluida y de la temperatura del sistema de adsorción

$a_{s p}^{\sigma}=f(p)_{T}$

A la Ecuación 5 se le denomina isoterma de adsorción del componente y es la ecuación fundamental para la descripción termodinámica de los estados de equilibrio del fenómeno de adsorción, a partir del comportamiento de cada componente en la fase fluida.

\section{ADSORCIÓN COMO FENÓMENO DE TRANSICIÓN DE FASE}

A diferencia del plano de Gibbs o GDS, en un sistema real como el que se ilustra en la Figura 1a, existe un gradiente de concentración a través de la capa de adsorción, razón por la cual ésta no se considerada como una fase. La conveniencia del sistema de referencia de Gibbs se hace evidente al entender la adsorción como un intercambio de materia entre dos fases, la fluida y la GDS, permitiendo de esta manera ver este proceso como la transición que experimentan los componentes del sistema desde una fase de tres dimensiones a una fase superficial de dos dimensiones, o simplemente como la pérdida de grados de libertad para el componente que se adsorbe.

En el desarrollo del formalismo termodinámico de la GDS se asume que el volumen del adsorbente es constante y que la superficie es homogénea e inerte, es decir que el potencial de adsorción es el mismo en toda la superficie y que ésta no modifica su área superficial, energía interna y entropía durante la adsorción física o fisisorción, de modo que

$$
\left(\frac{\partial G^{s}}{\partial n^{\sigma}}\right)_{T, A}=0
$$

Según la Ecuación 6 el equilibrio termodinámico para el sistema de adsorción queda definido de la siguiente manera

$$
\left(\frac{\partial G}{\partial n^{\sigma}}\right)_{T, p, A, n}=\left(\frac{\partial G^{\sigma}}{\partial n^{\sigma}}\right)_{T, A}+\left(\frac{\partial G^{f}}{\partial n^{\sigma}}\right)_{T, p}+\left(\frac{\partial G^{s}}{\partial n^{\sigma}}\right)_{T, A}=0
$$

en función de la temperatura, presión, área superficial del adsorbente y cantidad de componente.

Como ilustra la Figura 1b, la GDS se ubica en la superficie del adsorbente, de modo que el balance de componente durante la adsorción para el sistema cerrado, se puede escribir de la siguiente manera

$$
d n=d n^{\sigma}+d n^{f}=0
$$

Según lo anterior, en el equilibrio se cumplen las siguientes relaciones

$\left(\frac{\partial G^{\sigma}}{\partial n^{\sigma}}\right)_{T, A}=-\left(\frac{\partial G^{f}}{\partial n^{\sigma}}\right)_{T, p}=\left(\frac{\partial G^{f}}{\partial n^{f}}\right)_{T, p}$

Una vez más se aprecia la conveniencia del formalismo de Gibbs para el fenómeno de adsorción, ya que con lo planteado hasta el momento se llega a que en el equilibrio de la transición del componente, de una fase en tres dimensiones a una en dos dimensiones, se obedece la igualdad en los potenciales químicos de los componentes entre la fase adsorbida y la fluida, $\mu^{\sigma}=\mu^{f}$.

\section{Isoterma de adsorción de Gibbs}

El potencial termodinámico energía de Gibbs para la GDS, cuando la fase fluida es una mezcla binaria de $n_{l}$ moles de solvente y $n$, moles de soluto, se expresa en función de las correspondientes variables de estado: ${ }^{4}$ temperatura, presión, componentes y área superficial del adsorbente

$G^{\sigma}=G^{\sigma}\left(T, p, n_{1}, n_{2}, A\right)$

La diferencial total de la energía de Gibbs para la GDS se expresa mediante la siguiente ecuación

$d G^{\sigma}=-S^{\sigma} d T+V^{\sigma} d p+\sum_{i=1}^{2} \mu_{i}^{\sigma} d n_{i}^{\sigma}+\gamma^{\sigma} d A$

Después de tener en cuenta las siguientes relaciones, ${ }^{1,3,6}$

$\left(\frac{\partial G^{\sigma}}{\partial T}\right)_{p, n_{1}, n_{2}, A}=-S^{\sigma}$

$\left(\frac{\partial G^{\sigma}}{\partial p}\right)_{\tau, n_{1}, n_{2}, A}=V^{\sigma}$

$\left(\frac{\partial G^{\sigma}}{\partial n_{i}}\right)_{T, p, n_{j}, A}=\mu_{i}^{\sigma}$

$\left(\frac{\partial G^{\sigma}}{\partial A}\right)_{T, p, n_{1}, n_{2}}=\gamma^{\sigma}$

La Ecuación 15 introduce la cantidad intensiva tensión interfacial, correspondiente a la interfase sólido-fluido, que en el modelo de referencia de Gibbs seria la GDS, para cuantificar los cambios en la energía de Gibbs del sistema por cambios en el área superficial del adsorbente.

Después de aplicar a la Ecuación 11 el teorema de Euler y diferenciar se tiene, ${ }^{3,4}$

$d G^{\sigma}=\sum_{i=1}^{2} \mu_{i}^{\sigma} d n_{i}^{\sigma}+\sum_{i=1}^{2} n_{i}^{\sigma} d \mu_{i}^{\sigma}+A d \gamma^{\sigma}+\gamma^{\sigma} d A$

Al comparar las Ecuaciones 11 y 16, y teniendo en cuenta la Ecuación 2 se concluye que

$d \gamma^{\sigma}=-\sum_{i=1}^{2} \Gamma_{i} d \mu_{i}^{\sigma}$ 
La Ecuación 17 es una ecuación del tipo Gibbs-Duhem que muestra la relación entre las cantidades intensivas de las dos fases, a $\mathrm{T}$ y p constantes, sin una contribución significativa de la superficie. Cuando en la fase fluida la relación $\mathrm{n}_{2} / \mathrm{n}_{1}$ es pequeña, en el plano de Gibbs se cumple que $\Gamma_{1} \approx 0$, de modo que la Ecuación 17 queda finalmente como

$$
\Gamma_{2}^{(1)}=-\left(\frac{\partial \gamma^{\sigma}}{\partial \mu_{2}^{\sigma}}\right)_{T}
$$

A la cantidad $\Gamma_{2}^{(1)}$ se le denomina la concentración de exceso relativo del componente 2 con respecto al 1, y a la Ecuación 18 se le conoce como isoterma de adsorción de Gibbs. Para relacionar $\Gamma_{2}^{(1)}$ con cantidades medibles, recordamos la conclusión de la Ecuación 9 de igualdad del potencial químico del soluto entre la fase fluida y la GDS para el estado de equilibrio, y que la derivada del potencial químico con respecto al soluto es $d \mu^{\sigma}{ }_{2}=d \mu^{\mathrm{f}}{ }_{2}=\operatorname{RTdlna}_{2}^{\mathrm{f}}$, donde $\mathrm{a}_{2}^{\mathrm{f}}$ es para la actividad del soluto en la fase fluida. Estas consideraciones permiten escribir la Ecuación 18 de la siguiente manera

$$
\Gamma_{2}^{(1)}=-\frac{1}{R T}\left(\frac{\partial \gamma^{\sigma}}{\partial \ln a_{2}^{f}}\right)
$$

La Ecuación 19 obtenida desarrollando el formalismo termodinámico para el modelo de Gibbs, relaciona las cantidades de exceso que describen la adsorción con cantidades que se pueden medir o inferir a partir de datos experimentales, como son la tensión interfacial y la concentración. La dependencia de la tensión superficial con la concentración se establece experimentalmente, ya que no existe una ecuación de estado que las relacione. Así que de la pendiente positiva o negativa de la curva de tensión superficial en función de la concentración, se establece si hay enriquecimiento o agotamiento del soluto durante el proceso de adsorción.

\section{Ecuación de estado a partir de isotermas de adsorción: ley limite de Henry}

En la interfase sólido-líquido tiene lugar una presión expansiva, $\pi$, originada por la acumulación de moléculas de soluto en ésta durante la adsorción. Esta presión expansiva es naturalmente contrarrestada por la tendencia de la interfase a contraerse bajo fuerzas normales de tensión superficial, $\gamma^{\sigma}$, como lo muestra la siguiente relación: $\gamma^{\sigma}=\gamma_{0}^{\sigma}-\pi$, donde $\gamma_{0}^{\sigma}$ es la tensión superficial inicial del sólido.

La Ecuación 19 se modifica para incluir la presión expansiva de la interfase, quedando

$\Gamma_{2}^{(1)}=\frac{1}{R T}\left(\frac{\partial \pi}{\partial \ln a_{2}^{f}}\right)$

Para ilustrar la importancia de la Ecuación 20, haciendo uso de la ley límite de Henry, deduciremos la ecuación de estado que define la capa adsorbida de un gas (ó solución ideal diluida).

Para hacer una primera aproximación de un sistema real al formalismo anteriormente desarrollado para el sistema de referencia de Gibbs, convenientemente suponemos, a partir de la Ecuación 5, que la adsorción de un gas sobre un sólido se describe por una isoterma de la forma $a_{s p}^{\sigma}=f(p)_{T}=K p$ (experimentalmente verificable para un sistema a bajas presiones o infinitamente diluido), donde $K$ es la constante de proporcionalidad (o constante de Henry) y $p$ la presión, entonces la Ecuación 20 queda de la siguiente manera (para gas ideal la actividad está directamente relacionada con la presión), después de integrar desde cero para $p$ y $\pi$

$$
\pi=\frac{K R T}{a_{s p}} p
$$

Esta última ecuación se puede expresar de la forma

$\pi=\frac{k_{B} T}{\rho}$

donde $k_{B}$ es la constante de Boltzmann y $\rho$ es el área por molécula definida de la siguiente manera, $\rho=\frac{m^{s} a_{s p}}{n^{\sigma} N_{A}}$, donde $N_{A}$ es para el
número de avogrado.

La Ecuación 22 significa que cuando los estados de equilibrio de la adsorción se describen por la ley límite de Henry, la fase adsorbida se comporta como un gas ideal en una fase bidimensional $\left(\pi \rho=\mathrm{K}_{\mathrm{B}} \mathrm{T}\right)$.

\section{Isoterma de adsorción a partir de ecuaciones de estado: ${ }^{7}$ desviaciones de la idealidad}

Una segunda aproximación de un sistema real al formalismo de Gibbs se hace considerando que existen interacciones adsorbatoadsorbato, es decir interacciones moleculares entre las especies adsorbidas. Interacciones que implican desviaciones del comportamiento ideal de la capa adsorbida bidimensional.

Asumiendo que la ecuación de estado que define la capa adsorbida cuando hay interacciones intermoleculares, es de la siguiente forma

$\pi\left(\rho-\rho^{0}\right)=k_{B} T$

donde $\rho^{0}$ hace referencia al área ocupada por la molécula sobre la superficie en ausencia de interacciones moleculares, y $\rho$ es para el área ocupada por cada molécula en presencia de las interacciones, la cual es función de la cantidad de exceso adsorbida. Al incorporar en la Ecuación 20 la definición dada en la sección anterior para el área molecular, y reemplazando en ésta la diferenciación de la Ecuación 23 con respecto a $\rho$, se llega a la siguiente expresión

$-\left(\frac{\rho}{\left(\rho-\rho^{0}\right)^{2}}\right) d \rho=d \ln p$

Después de integrar indefinidamente la Ecuación 24, se obtiene

$\ln \left(\frac{\theta}{1-\theta}\right)+\frac{\theta}{1-\theta}=\ln p+C$

donde $\rho / \rho^{0}=1 / \theta$. La variable $\theta$ se denomina la fracción de recubri-

miento, la cual es igual a la cantidad adsorbida dividida por la máxima cantidad que se debe adsorber para cubrir totalmente la superficie del

adsorbente, formando lo que se denomina una monocapa, $\theta=n^{\sigma} / n_{m}^{s}$.

Estrictamente en la definición de $\theta$ deberíamos, en lugar de $n^{\sigma}$, incluir

una cantidad $n^{s}$ que haga referencia a la cantidad real adsorbida.

Reorganizando la Ecuación 25, con el propósito de eliminar el logaritmo, legamos a la siguiente expresión

$K^{\prime} p=\left(\frac{\theta}{1-\theta}\right) e^{\left(\frac{\theta}{1-\theta}\right)}$ 
donde $\operatorname{In} K^{\prime}=\mathrm{C}, C$ es la constante de integración de la Ecuación 25.

Teniendo en cuenta que a presiones muy bajas la fracción de recubrimiento es cercana a cero, la exponencial de la Ecuación 26 converge rápidamente a cero, así que esta ecuación queda finalmente de la siguiente manera

$$
K^{\prime} p=\frac{\theta}{1-\theta}
$$

La Ecuación 27 se puede reorganizar para darle la forma de la isoterma de Langmuir

$\theta=\frac{p K^{\prime}}{1+p K^{\prime}}$

A muy bajas presiones, en el límite de gas ideal, la Ecuación 28 se reduciría a la ley de Henry, así que su validez se restringe a la vecindad de este comportamiento límite.

\section{ENERGÍAS INVOLUCRADAS EN LA ADSORCIÓN}

La interpretación de la adsorción como un proceso de transición de un componente, desde una fase en tres dimensiones a una en dos dimensiones, permite analizar cualitativamente los cambios en los potenciales termodinámicos, energía de Gibbs, entropía y entalpía. Debido a ésta transición se reduce el desequilibrio de fuerzas atractivas que existen en una superficie y por tanto, la energía libre superficial de un sistema heterogéneo disminuye, soportando la espontaneidad del proceso. Igualmente esta transición, por pérdida en el número de grados de libertad de los componentes adsorbidos, va acompañada de la disminución en la entropía. Así que haciendo uso de la siguiente ecuación fundamental, $\Delta_{\text {ads }} \mathrm{G}=\Delta_{\text {ads }} \mathrm{H}-\mathrm{T} \Delta_{\text {ads }} \mathrm{S}$, se llega a que el $\Delta_{\text {ads }} \mathrm{H}$ de adsorción debe ser siempre negativo.

En el estudio cuantitativo de la energía de adsorción, usualmente se utiliza uno de los dos siguientes métodos: el isostérico y la calorimetría de inmersión. ${ }^{1,9}$

\section{Método isostérico}

Este método es útil para estimar la entalpía diferencial estándar de adsorción, bajo la restricción de fracción de recubrimiento constante de la superficie.

Recordando que para un sistema en equilibrio termodinámico se satisface la siguiente ecuación fundamental, ${ }^{10}$

$$
\Delta G_{a d}^{0}=-R T \ln K_{e q}=\Delta H_{a d}^{0}-T \Delta S_{a d}^{0}
$$

donde $\mathrm{K}_{\mathrm{eq}}$, es para la constante de equilibrio dinámico, tipo equilibrio químico, como es el caso del equilibrio establecido por transferencia de componentes entre las fases fluida y la GDS durante la adsorción. De esta manera la constante $K^{\prime}$ de la isoterma de Langmuir, Ecuación 28 , se relaciona directamente con la constante de equilibrio de la Ecuación 29, en la vecindad de la ley limite de Henry. Incorporando la isoterma de Langmuir en la Ecuación 29 obtenemos

$$
\Delta G_{a d}^{0}=-R T \ln \left(\frac{\theta}{(1-\theta)} \frac{1}{p}\right)=\Delta H_{a d}^{0}-T \Delta S_{a d}^{0}
$$

Asumiendo que la entalpía diferencial estándar y la entropía estándar de la Ecuación 30 son aproximadamente constantes en un intervalo estrecho de temperatura, la diferencial de la Ecuación 30 con respecto a la temperatura, a fracción de recubrimiento constante, es

$$
\left[\frac{\partial}{\partial T}(\ln p)\right]_{\theta}=-\frac{\Delta H_{a d}^{0}}{R T^{2}}
$$

La Ecuación 31 es una ecuación tipo Clausius-Clapeyron, para la cual se tienen unas condiciones bien definidas en términos de la magnitud de la presión (ó concentración de una solución), del intervalo de temperatura y de restricciones en las estructuras de la fases fluida y adsorbida, las cuales no se pueden alterar durante su aplicación.

Para relacionar directamente la entalpía diferencial estándar de adsorción a fracción de recubrimiento constante ó entalpía isostérica de adsorción, con el calor involucrado en la adsorción, la capacidad de monocapa, $\mathrm{n}_{\mathrm{m}}^{\mathrm{s}}$, debe ser constante en función de la temperatura, como se aprecia en la siguiente ecuación, ${ }^{8}$

$$
\Delta H_{a d}=Q+\delta \frac{R T^{2}}{1-\theta}
$$

donde $\delta=\frac{1}{n_{m}^{s}}\left[\frac{\partial n_{m}^{s}}{\partial T}\right]$. Las consideraciones realizadas para llegar a la Ecuación 32 se pueden consultar en la referencia 8. La Figura 2 muestra que la capacidad de monocapa es función de la temperatura. Si no se tiene en cuenta que tanto puede variar la capacidad de monocapa con la temperatura, se pueden llegar a conclusiones erróneas con respecto a la exotermicidad del proceso, como se aprecia en la Figura 3.

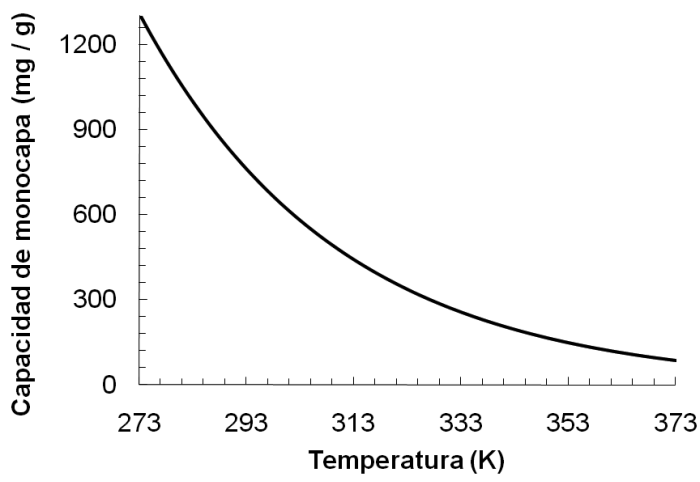

Figura 2. Dependencia de la capacidad de monocapa con respecto a la temperatura, para la adsorción en un sistema de un soluto en un solvente en contacto con un adsorbente microporoso, calculada a partir de la siguiente ecuación empírica deducida de evidencias experimentales, $n_{m}^{s}(T)=\exp \left[\chi\left(1-\frac{T}{T^{0}}\right)\right]$ donde $n_{m, 0}^{s}$ es la capacidad de monocapa a una temperatura de referencia $T^{0}$ y $\chi$ es una constante adimensional ${ }^{8}$

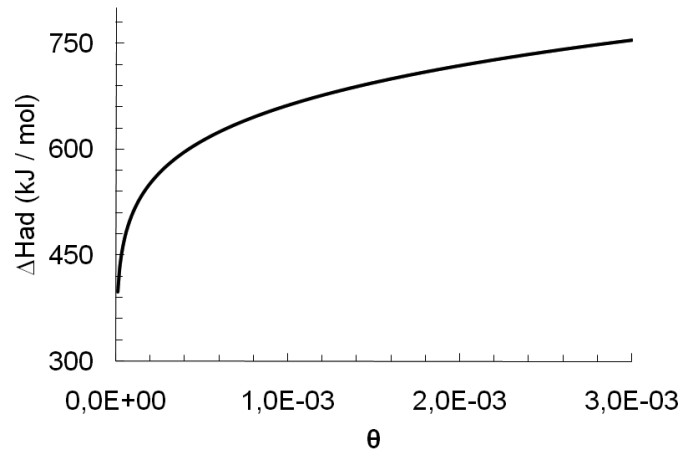

Figura 3. Entalpía isostérica en función de la fracción de recubrimiento $(\theta)$, para el proceso de adsorción de un soluto en contacto con un adsorbente microporoso, usando la ecuación tipo Clausius-Clapeyron (31). El valor positivo de la entalpía isostérica de adsorción indicaría un proceso endotérmico 
Cuando los datos experimentales no se ajustan adecuadamente a la descripción de una isoterma tipo Langmuir, es posible que al utilizar esta en el cálculo de la entalpía isostérica también se lleguen a conclusiones erróneas. Existe una gran variedad de propuestas de isotermas de adsorción, entre las que destacamos la isoterma de Tóth, Ecuación 33, por considerar la dependencia de la capacidad de monocapa con la temperatura, y una constante empírica que también es función de la temperatura,

$$
n^{s}=n_{m}^{s} \frac{K p}{\left[1^{\prime}+(K p)^{t}\right]^{1 / t}}
$$

donde $\mathrm{n}^{\mathrm{s}}$ es la cantidad adsorbida, $p$ la presión de equilibrio, $K$ ' una constante característica de la adsorción y $t$ un parámetro relacionado con la heterogeneidad del sistema. Los parámetros $t$ y $\mathrm{n}_{\mathrm{m}}^{\mathrm{s}}$ dependen empíricamente de la temperatura de la siguiente manera

$$
t(T)=t_{0}+\alpha\left(1-\frac{T_{0}}{T}\right)
$$

$\mathrm{y}$

$$
n_{m}^{s}(T)=n_{m 0}^{s} e^{\chi\left(1-\frac{T}{T_{0}}\right)}
$$

donde $t_{0}$ es el valor de $t$ a una temperatura de referencia $T_{0} \alpha$ y $\chi$ son constantes adimensionales y $\mathrm{n}_{\mathrm{m} 0}^{\mathrm{s}}$ la capacidad de monocapa a una temperatura de referencia $T_{0}$.

Al reemplazar la isoterma de Tóth en la Ecuación 31 se obtiene que la entalpía isostérica diferencial de adsorción queda expresada de la siguiente manera, ${ }^{8}$

$$
\Delta H_{a d}=Q+\frac{1}{t} \alpha R T_{0}\left[\ln \left(\frac{\theta}{\left(1-\theta^{t}\right)^{1 / t}}\right)-\frac{\ln \theta}{1-\theta^{t}}\right]
$$

La Ecuación 36 relaciona directamente la entalpía isostérica de adsorción con el calor involucrado en el proceso cuando la fracción de recubrimiento tiende a cero. La Figura 4 muestra que la entalpía isostérica de adsorción es exotérmica en función de la fracción de recubrimiento.

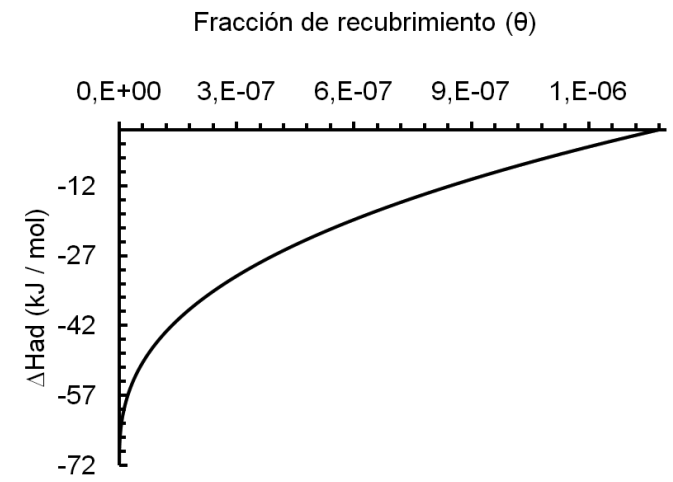

Figura 4. Entalpía isostérica exotérmica en función de la fracción de recubrimiento $(\theta)$, para el proceso de adsorción de un soluto en contacto con un adsorbente microporoso, según la Ecuación 36

\section{Calorimetría de inmersión}

Con este método se busca determinar directamente la entalpía de adsorción, sin hacer referencia ni a un estado estándar y ni a una isoterma de adsorción. En una primera aproximación a este método el sólido bajo vacio es inmerso en un líquido puro, a una temperatura y presión determinadas. ${ }^{11}$ La entalpía medida por este método se denomina entalpía de inmersión, y se puede asociar a cambios en el área superficial del sólido ó a la variación en la tensión interfacial. Sin embargo es importante aclarar que en estos métodos, normalmente el área superficial del sólido se asume constante durante el proceso de inmersión. La entalpía de inmersión por unidad de área se describe habitualmente por la ecuación,,$^{1,11}$

$-\Delta_{i m m} H=\left(\gamma_{S}^{o}-\gamma_{S L}\right)-T\left(\frac{\partial\left(\gamma_{S}^{o}-\gamma_{S L}\right)}{\partial T}\right)_{p}$

donde $\gamma_{\mathrm{S}}^{0}$ es para la tensión superficial del sólido en el vacío y $\gamma_{\mathrm{SL}}$ para la interfase sólido-líquido. La Ecuación 37 se obtiene al analizar el cambio de entalpía en el sólido, cuando pasa de estar seco (en vacio) a estar húmedo. La situación es un poco más compleja cuando una cantidad dada de líquido se vaporiza desde esta fase y se adsorbe sobre la superficie del sólido. La variación en la entalpía de este sistema se define como entalpía de mojado de inmersión por unidad de área, ${ }^{11}$

$-\Delta_{w} H=\left(\gamma_{S}^{o}-\gamma_{S L}\right)-T\left(\frac{\partial\left(\gamma_{S}^{o}-\gamma_{S L}\right)}{\partial T}\right)_{p}$

donde $\gamma_{\mathrm{SV}}^{\mathrm{P}_{\mathrm{L}}}$ es la tensión interfacial sólido-vapor, para algunos valores de presión parcial del líquido $p_{L}$.

Cuando un líquido se coloca sobre la superficie de un sólido, previamente en contacto con la fase de vapor, éste puede tender a "expandirse" sobre la superficie del sólido si la tensión expansiva, $\sigma^{\mathrm{SLV}}$, definida como $\sigma^{\mathrm{SLV}}=\gamma^{\mathrm{SV}}-\gamma^{\mathrm{LV}}-\gamma^{\mathrm{SL}}$, es positiva (esta tensión también se puede señalar como el trabajo de expansión del líquido sobre el sólido por unidad de área)., ${ }^{2,7}$

La relación de Young describe la posición de equilibrio cuando se coloca una gota sobre una superficie sólida plana y horizontal. Si se considera el caso general de una gota de un líquido sobre un sólido, en equilibrio con su fase de vapor, la relación de Young para este sistema se expresa como, ${ }^{4,7}$ (Figura 5)

$\gamma^{L V} \cos \theta=\gamma^{S V}-\gamma^{S L}$

Al sustituir la Ecuación 39 de Young en la Ecuación 38 y además, asumiendo un mojado perfecto del líquido a la superficie (ángulo de contacto cero), la entalpía de mojado de inmersión por unidad de área se reduce $\mathrm{a},{ }^{1,11}$

$-\Delta_{w} H=\gamma_{L V}-T\left(\frac{\partial\left(\gamma_{L V}\right)}{\partial T}\right)_{p}=H_{L}$

donde $H_{L}$ es la entalpía superficial del líquido. Esta última ecuación se puede escribir como $-\Delta_{w} \mathrm{H}^{*}=\mathrm{a}_{\mathrm{sp}} \mathrm{H}_{\mathrm{L}}$, donde $a_{s p}$ es el área de superficie

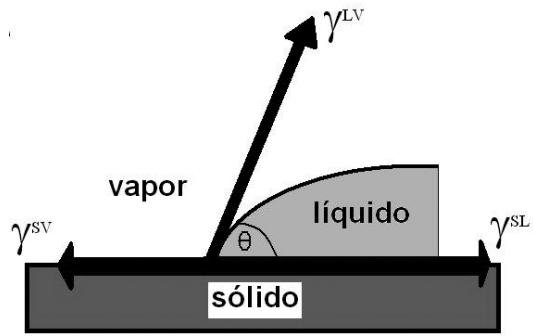

Figura 5. Esquema general de los diferentes componentes de la ecuación de Young, cuando una gota se coloca sobre una superficie sólida plana y horizontal 
específica del sólido. Esta relación ha sido discutida ampliamente por Gregg y Sing, puesto que es una alternativa al método BET (BrunauerEmmett-Teller) para determinar áreas superficiales de sólidos. ${ }^{1,11}$

Al restar la Ecuación 38 de la 37, establecemos como se relaciona la entalpía de adsorción con la de mojado y la de inmersión, ${ }^{11}$

$\Delta_{a d s} H=\Delta_{i m m} H-\Delta_{w} H$

\section{CONCLUSIONES}

La contribución más sobresaliente de G. W Gibbs para la descripción termodinámica de la adsorción, es la consideración de que este fenómeno tiene lugar en un plano imaginario que permite ver la zona de adsorción como una fase. A partir de este modelo el fenómeno de adsorción se puede entender como un proceso de transferencia de componente entre fases, el cual queda bien definido en el equilibrio por la igualdad del potencial químico de los componentes entre las fases involucradas. Si bien es un modelo simplificado o idealizado, permite desarrollar con rigurosidad el formalismo termodinámico que describe la adsorción. Así, partiendo de una ecuación de estado generalizada, como la Ecuación 10 deducimos la isoterma de adsorción de Gibbs, ó partiendo de una ecuación de estado como la 23 deducimos la isoterma de Langmuir. Igualmente, el formalismo permite que a partir de considerar una determinada isoterma de adsorción, como es la ley límite de Henry, se pueda deducir una ecuación de estado como la 22. Otro de los aspectos a destacar del formalismo termodinámico de Gibbs para la adsorción, es la puesta en evidencia de las restricciones que hay que imponer al sistema, como son bajas presiones o soluciones muy diluidas, para que la ecuación de estado y la isoterma de adsorción describan apropiadamente un sistema real.

A diferencia de la determinación directa de la entalpía de adsorción por calorimetría de inmersión, en la cuantificación de ésta por el método isostérico quedan en claro los siguientes aspectos: que ésta realmente hace referencia a una entalpía diferencial estándar de adsorción y que para correlacionarla con la entalpía real de adsorción, su estimación debe hacerse en un intervalo estrecho de temperatura, y que el ajuste de los datos a una isoterma no apropiada puede llevar a conclusiones erróneas con respecto a la magnitud y la exotermicidad de la adsorción.

\section{AGRADECIMIENTOS}

A la Dirección Nacional de Investigaciones de la Universidad Nacional de Colombia por la financiación a través del proyecto 20601002443. J. M. Arias y E. Paternina agradecen a la Universidad Nacional de Colombia por el apoyo económico brindado a través del cargo auxiliar docente.

\section{REFERENCIAS}

1. Rouquerol, F.; Rouquerol, J.; Sing, K.; Adsorption by powders \& porous solids: principles, methodology and applications, Academic Press: London, 1999.

2. IUPAC; Manual of symbols and terminology for physicochemical quantities and units: definitions, terminology and symbols in colloid and surface chemistry, IUPAC: Washington, 2001.

3. Tóth, J.; Adsorption: Theory, modeling and analysis, Marcel Dekker: New York, 2002.

4. Turmine, M.; Letellier, P.; J. Colloid Interface Sci. 2000, 227, 71.

5. Myers, A.; AIChE J. $2002,48,145$.

6. Kondepudi, D.; Prigogine, I.; Modern thermodynamics: from heat engine to dissipative structures, John Wiley: Chichester, 1998.

7. Hiemenz, P. C.; Rajagopal, R.; Principles of colloid and surface chemistry, $3^{\text {rd }}$ ed., Marcel Dekker: New York, 1997.

8. Do, D.; Adsorption Analysis: equilibria and kinetics, Imperial College Press: London, 1998.

9. Adamson, A.; Gast, A.; Physical Chemistry of Surfaces, $6^{\text {th }}$ ed., Wiley: New York, 1997.

10. Attard, G.; Barnes, C.; Surfaces, Oxford University Press: Oxford, 1998.

11. Douillard, J. M.; J. Colloid Interface Sci. 1996, 182, 308.

12. Silvestre-Albero, J.; Gómez de Salazar, C.; Sepúlveda-Escribano, A.; Rodríguez-Reinoso, F.; Colloids Surf. A: Physicochem. Eng. Aspects 2001, 187-188, 151. 